\title{
Reversible Laser Annealing and Magneto-Optical Characterization of HTSC Thin Films
}

\author{
J. Eisenmenger, J. Zimmermann, J. Schiessling, U. Bolz, B.-U. Runge, \\ and $\mathrm{P}$. Leiderer
}

Universität Konstanz, Fakultät für Physik, D-78457 Konstanz, Germany

\begin{abstract}
Summary: The oxygen content in YBCO thin films can be changed locally by scanning a focused laser beam across the film surface at defined oxygen partial pressure. In contrast to annealing experiments in a furnace where the oxygen concentration remains homogeneous in the direction of the film layer, the local heating with a laser beam leads also to a lateral oxygen diffusion in the thin film. By investigating the laser written structures a deeper understanding of the patterning process itself and the oxygen diffusion in $\mathrm{YBCO}$ thin films could be obtained. For the detection of laser written structures and natural defects in HTSC thin films the magneto-optical method is highly qualified. It allows to visualize the magnetic screening behaviour with a high lateral resolution in the range of micrometers. In the past this technique was used to investigate the magnetic flux distribution in smaller samples at temperatures far below the boiling point of liquid nitrogen. In this work the magnetic resolution of the method was optimized so that much weaker magnetic field contrasts at $77 \mathrm{~K}$ can still be detected. In particular the presented apparatus allows the characterization of double-sided 3" HTSC Wafers even under conditions where cooling with liquid helium or closed-cycle refrigerators is not available.
\end{abstract}

\section{Reversible Laser Annealing}

\subsection{Introduction}

Structures of the size of micrometers play an important role at the HTSC thin film device production. Several techniques are suitable for the patterning of YB$\mathrm{CO}$ films such as wet chemical and plasma etching, ion milling and laser ablation. The reversible laser annealing is an additional technique that allows to fabricate structures in the micrometer range. It is based on the observation that the electrical and optical properties of $\mathrm{YBa}_{2} \mathrm{Cu}_{3} \mathrm{O}_{6+\mathrm{x}}$ are very sensitive to its oxygen content. In the orthorhombic phase (i.e. $0.4<x<1$ ) $\mathrm{YBa}_{2} \mathrm{Cu}_{3} \mathrm{O}_{6+\mathrm{x}}$ is superconducting and behaves above $T_{c}$ like a metallic conductor concerning the electronic 


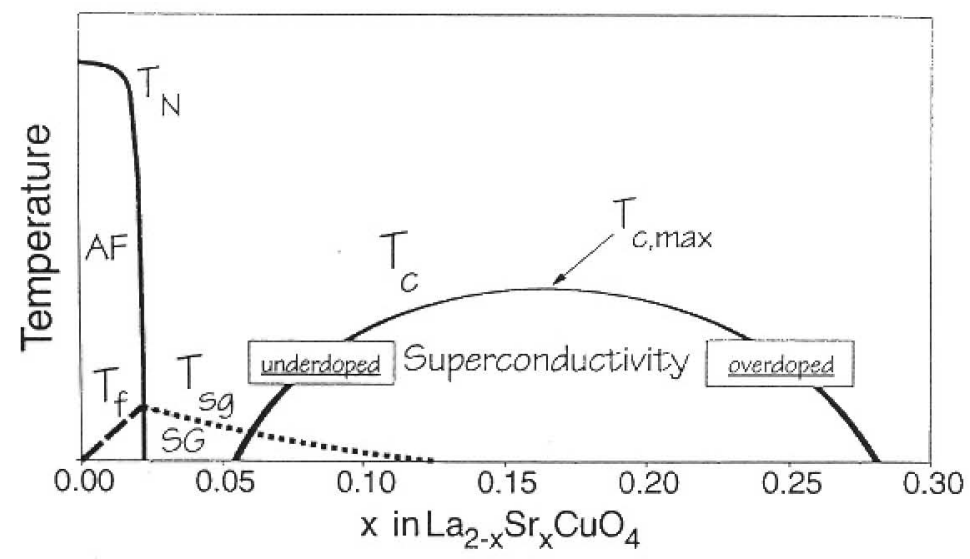

Figure 1 Schematic representation of the doping-dependent phase diagram for $\mathrm{La}_{2}-x \mathrm{Sr}_{x} \mathrm{CuO}_{4} . \mathrm{AF}$ and $\mathrm{SG}$ denote the antiferromagnetic and spin glass phases.

transition coinciding with the onset of superconductivity and $T_{c}$ reaches its maximum value of about $38 \mathrm{~K}$ at optimal doping $\mathrm{x} \approx 0.16$. As also shown in the figure, at intermediate doping levels extending from the Néel state and well into the superconducting regime, short-rangéd AF fluctuations survive and at low temperatures freeze into a disordered spin-glass like state (annotated SG) which coexists with superconductivity.

In this article, we will show that the above phase diagram appears to be generic to all HTS cuprates. We will discuss the nature of the magnetic state in the low doping regime $(\mathrm{p}<0.1)$ in $\mathrm{La}_{2-x} \mathrm{Sr}_{x} \mathrm{CuO}_{4}$ and $\mathrm{Y}_{1-x} \mathrm{Ca}_{x} \mathrm{Ba}_{2} \mathrm{Cu}_{3} \mathrm{O}_{6}$ and will show that in both systems an electronically non uniform state evolves upon doping, which is reasonably well described by the concept of electronic phase separation into hole rich and hole poor regions $[1,2,3,4]$.

\section{Principle of $\mu \mathrm{SR}$}

The technique of muon spin rotation or relaxation $(\mu \mathrm{SR})$ is a powerful tool for studying internal magnetic fields within solids [5]. In the context of high temperature superconducting (HTS) cuprates $\mu \mathrm{SR}$ experiments have provided important contributions to a better understanding of the physics of the vortex state, superfluid density and the complex interplay between magnetism and superconductivity that distinguishes the HTS cuprates (see e.g. reference [6]).

The basic idea of a $\mu \mathrm{SR}$ experiment is very similar to that of the NMR technique. Positive muons are incorporated as local probes in the host lattice of the sample to be studied. The spin of the muon and the related magnetic moment act as a sensitive probe for the local magnetic field through its precession in the field with a frequency of $\omega_{\mu}=\gamma_{\mu} B_{\mu}$ where $\gamma_{\mu}=851.4 \mathrm{MHz} / \mathrm{T}$ is the gyromagnetic 


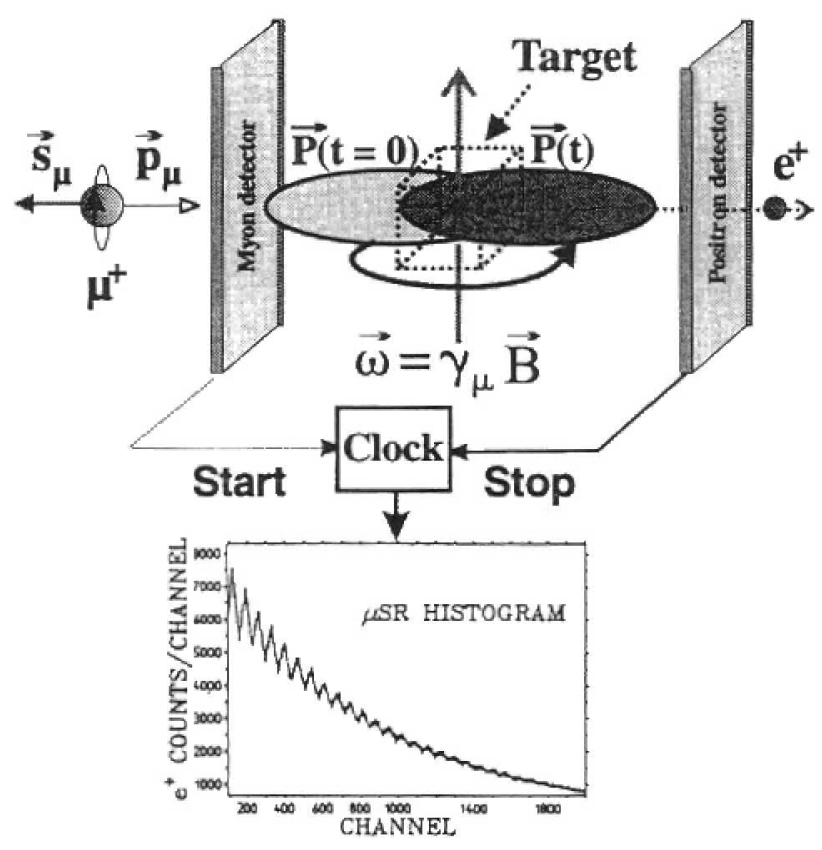

Figure 2 Schematic diagram showing the main components of the $\mu \mathrm{SR}$ technique.

ratio of the muon and $\mathrm{B}_{\mu}$ is the local field at the muon site. The polarisation of the muon is conveniently determined by the fact that when a muon decays (half life $2.2 \mu \mathrm{s}$ ) the resulting positron is emitted preferentially in the direction of the instantaneous polarisation. A schematic diagram of the standard $\mu \mathrm{SR}$ experiment is shown in Fig. 2. A beam of $100 \%$ spin-polarised muons is directed onto the cuprate sample which may be a sintered polycrystalline body, an oriented single crystal, a mosaic of single crystals or even a compact of powder. The injected muons thermalise rapidly without any significant loss in polarisation and come to rest at distinct sites in the crystallographic unit cell, forming a muoxyl bond with apical or chain oxygens [7].

A fascinating aspect of the $\mu \mathrm{SR}$ technique compared to other microscopic techniques, is the possibility of measuring internal magnetic fields in zero external field. The observation of a muon spin precession signal in zero field is an unambiguous indication for the existence of a magnetic hyperfine field at the muon site, which is produced by the magnetic moments of the surrounding atoms and has to be static or slowly fluctuating on a timescale of microseconds in order to be seen by the muon. In the high- $\mathrm{T}_{c}$ cuprates the internal field at the muon site is dominated by dipolar contributions from the surrounding moments and due to the $\mathrm{r}^{-3}$ dependence of the dipolar coupling the dominant contributions come from dipoles within the first and second coordination shells. Thus another advantage of the experiment is the sensitivity of the muon probe to extremely short ranged magnetic correlations on a nanometer scale. 


\section{Sample Preparation}

Systematic muon spin rotation studies so far focused on the $\mathrm{La}_{2-x} \mathrm{Sr}_{x} \mathrm{CuO}_{4}[8]$ and $\mathrm{YBa}_{2} \mathrm{Cu}_{3} \mathrm{O}_{7-\delta}[9]$ systems. In $\mathrm{YBa}_{2} \mathrm{Cu}_{3} \mathrm{O}_{7-\delta}$ the phase diagram has to be drawn versus oxygen content and a reliable determination of $\mathrm{p}$ is difficult due to the rather complicated charge transfer from the $\mathrm{CuO}$ chains to the $\mathrm{CuO}_{2}$ planes. The $\mathrm{Y}_{1-x} \mathrm{Ca}_{x} \mathrm{Ba}_{2} \mathrm{Cu}_{3} \mathrm{O}_{6}$ system, i.e. with $\delta=1.0$, avoids this complication, because hole doping is achieved by the substitution of $\mathrm{Y}^{3+}$ by $\mathrm{Ca}^{2+}$. This allows one to directly control the hole concentration in the $\mathrm{CuO}_{2}$ planes in a quantitative manner and $\mathrm{p}=\mathrm{x} / 2$.

The polycrystalline samples were prepared by standard solid-state reaction methods using high purity powders. The doping state $\mathrm{p}$ was determined by measurements of the thermo-electric power (TEP) as described in reference [10]. The $\mathrm{T}_{c}$ values were obtained from measurements of the $\mathrm{AC}$-susceptibility and those values quoted in this article are the diamagnetic onsets extrapolated from the steepest diamagnetic part of the $\chi(T)$ curve. These $T_{c}$ values are up to $4 \mathrm{~K}$ below the onset temperatures for the most underdoped samples.

In the case of $\mathrm{Y}_{1-x} \mathrm{Ca}_{x} \mathrm{Ba}_{2} \mathrm{Cu}_{3} \mathrm{O}_{6}$ a series of sintering steps were applied to avoid a partial substitution of $\mathrm{Ca}$ on the $\mathrm{Ba}$ site. Stoichiometric precursors made from decomposing a mixture of $\mathrm{Y}_{2} \mathrm{O}_{3}, \mathrm{CaCO}_{3}, \mathrm{Ba}\left(\mathrm{NO}_{3}\right)_{2}$ and $\mathrm{CuO}$ were repeatedly reacted in air, then ground, milled and die-pressed and reacted starting from $910^{\circ} \mathrm{C}$ and increasing the temperature in $10 \mathrm{~K}$ steps up to $970^{\circ} \mathrm{C}$. If the final sintering temperature was too low energy-dispersive analysis by $\mathrm{x}$-rays (EDX) indicated Ba-rich impurity phases and the fully oxidized samples were less overdoped. Sintering at very high temperatures resulted in a Ca-rich impurity phase. To find the phase-pure window between these extremes the sintering temperature was progressively increased with intermediate grinding until Ba-rich phases were eliminated but before Ca-rich phases appeared. By annealing the samples at $770^{\circ} \mathrm{C}$ in $0.2 \%$ oxygen mixed with nitrogen then quenching into liquid nitrogen, oxygen deficiencies of the order of 0.95 were achieved and with further annealing at $550^{\circ} \mathrm{C}$ in a vacuum of about $10^{-7}$ torr $\delta$ was increased to 0.98 , as confirmed by neutron-diffraction structural refinement [11].

\section{$4 \quad \mu \mathrm{SR}$ Results and Discussion}

Representative $\mathrm{ZF}-\mu \mathrm{SR}$ time spectra are shown in Fig. 3. From this figure it is obvious that for a given hole concentration the time evolution of the muon spin polarization is identical for the $\mathrm{Y}_{1-x} \mathrm{Ca}_{x} \mathrm{Ba}_{2} \mathrm{Cu}_{3} \mathrm{O}_{6}$ and $\mathrm{La}_{2-x} \mathrm{Sr}_{x} \mathrm{CuO}_{4}$ system. The damped oscillations in the time spectrum of the $\mathrm{Y}_{0.94} \mathrm{Ca}_{0.06} \mathrm{Ba}_{2} \mathrm{Cu}_{3} \mathrm{O}_{6}$ sample together with the high transition temperature of $\mathrm{T}_{N} \sim 180 \mathrm{~K}$ implies that this sample exhibits $3 \mathrm{D}$ long range antiferromagnetic order. A strongly 


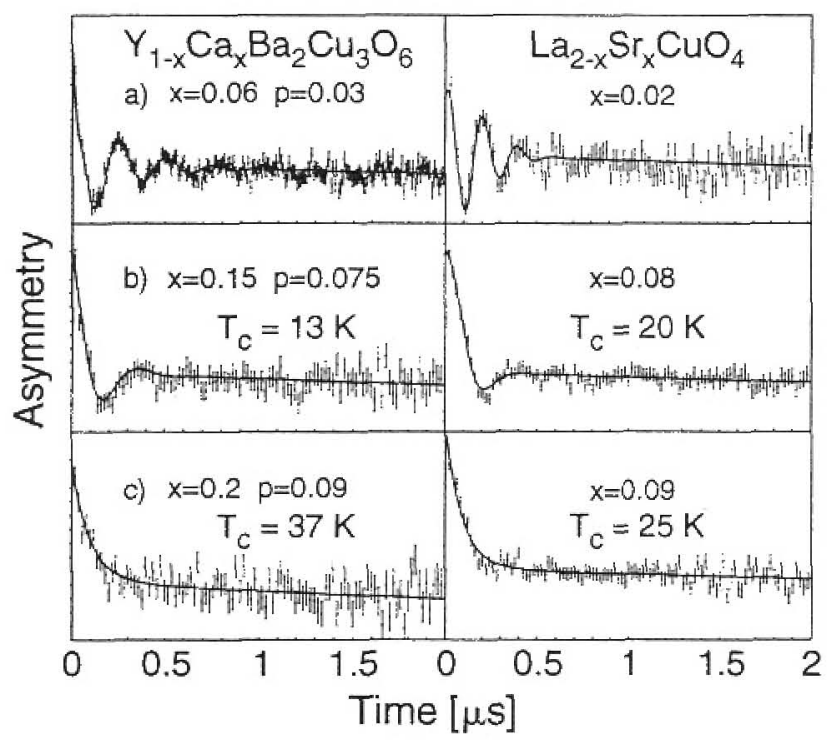

Figure $3 \mathrm{ZF}-\mu \mathrm{SR}$ spectra obtained at low temperatures ( $\mathrm{T}<1 \mathrm{~K}$ ) for various degrees of hole doping in $\mathrm{Y}_{1-x} \mathrm{Ca}_{x} \mathrm{Ba}_{2} \mathrm{Cu}_{3} \mathrm{O}_{6}$ and $\mathrm{La}_{2-x} \mathrm{Sr}_{x} \mathrm{CuO}_{4}$.

overdamped frequency is observed at low temperatures for $\mathrm{x}=0.15$, a superconducting sample with $\mathrm{T}_{c}=13 \mathrm{~K}$. Even for $\mathrm{Y}_{0.8} \mathrm{Ca}_{0.2} \mathrm{Ba}_{2} \mathrm{Cu}_{3} \mathrm{O}_{6}\left(\mathrm{~T}_{c}=37 \mathrm{~K}\right)$ a fast relaxing signal is observed, indicating the presence of strong magnetic correlations coexisting with superconductivity. The curves in Fig. 3 are the fit to the data using the following Ansatz for the time evolution of the muon spin polarization:

$$
G_{z}(t)=\frac{2}{3} \cos \left(\gamma_{\mu} B_{\mu} t\right) \exp \left(-\frac{1}{2}\left(\gamma_{\mu} \Delta B_{\mu} t\right)^{2}\right)+\frac{1}{3} \exp (-\lambda t)
$$

where $\gamma_{\mu}=851.4 \mathrm{MHZ} / \mathrm{T}$ is the gyromagnetic ratio of the muon, $\mathrm{B}_{\mu}$ the average internal magnetic field at the muon site and $\Delta \mathrm{B}_{\mu}$ its rms deviation. The two terms arise from the random orientation of the local magnetic field in a polycrystalline sample, which on average points parallel (perpendicular) to the muon spin direction with probability $1 / 3(2 / 3)[5]$.

In analogy to NMR a slowing down of magnetic fluctuations typically causes a maximum of $1 / T_{1}(=\lambda$ in the $1 / 3$ part of eq. 4.1$)$ at $\omega_{\mu} \tau_{c} \approx 1$, where $\omega_{\mu}$ is the $\mu^{+}$Zeeman frequency, and $\tau_{c}$ the average correlation time of the fluctuating transverse field components. A precessing $2 / 3$ component indicates static magnetic order on the time scale of the $\mu \mathrm{SR}$ technique $\left(\tau_{c}<10^{-6} \mathrm{~s}\right)$. For $\mathrm{p}>0.08$ no oscillations were observed and the $2 / 3$ part of $\mathrm{G}_{z}(t)$ was better represented by a relaxation of the form $\exp (-\Lambda \mathrm{t})$, which may indicate either a very strongly disordered static field distribution or rapid fluctuations. 


\subsection{Freezing of the doped holes in the low doping regime}

As an example for the behaviour of an only lightly doped system we discuss the data on $\mathrm{Y}_{0.95} \mathrm{Ca}_{0.05} \mathrm{Ba}_{2} \mathrm{Cu}_{3} \mathrm{O}_{6}$ which are displayed in Fig. 4. Well below the 3D Néel temperature of $\mathrm{T}_{N} \sim 225 \mathrm{~K}$ a second magnetic transition occurs at a temperature $\mathrm{T}_{f} \sim 20 \mathrm{~K}$. This is evident from the peak in the longitudinal relaxation rate $1 / T_{1}$ and the upturn of the muon spin precession frequency. $A$ corresponding transition within the AF state has been reported recently from La-NQR [12] and $\mu \mathrm{SR}$ studies [13] on $\mathrm{La}_{2-x} \mathrm{Sr}_{x} \mathrm{CuO}_{4}$ where $\mathrm{T}_{f}=815 \mathrm{~K} \cdot \mathrm{p}$ has been obtained for $\mathrm{p}<0.02$. This transition was ascribed to a freezing of the spins of the doped holes into a spin glass state which is superimposed on the preexisting 3D AF long-range order of the $\mathrm{Cu}^{2+}$ spins. Interestingly, we find that the spin freezing temperature $\mathrm{T}_{f}$ exhibits the same linear dependence on the planar hole content for $\mathrm{Y}_{1-x} \mathrm{Ca}_{x} \mathrm{Ba}_{2} \mathrm{Cu}_{3} \mathrm{O}_{6}$ and $\mathrm{La}_{2-x} \mathrm{Sr}_{x} \mathrm{CuO}_{4}$ (see Fig. 5a). According to the model of Gooding et al. [14], in which $J_{e f f} \cdot p \approx k_{B} T_{f}$, this implies that the effective in plane exchange coupling constant, $J_{e f f}$, is identical for both systems and that the freezing of the spin degrees of freedom is a property of the hole dynamics within a single plane. The Néel-state, however, persists to higher hole content in $\mathrm{Y}_{1-x} \mathrm{Ca}_{x} \mathrm{Ba}_{2} \mathrm{Cu}_{3} \mathrm{O}_{6}$ as compared to $\mathrm{La}_{2-x} \mathrm{Sr}_{x} \mathrm{CuO}_{4}$. This suggests that the bilayer coupling makes the $3 \mathrm{D}$ AF-state more robust to the presence of doped holes. A similar result was reported from a ${ }^{89} \mathrm{Y}$ NMR study of $\mathrm{T}_{N}$ in $\mathrm{Y}_{1-x} \mathrm{Ca}_{x} \mathrm{Ba}_{2} \mathrm{Cu}_{3} \mathrm{O}_{6}[15]$.

\subsection{Coexistence of antiferromagnetism and superconductivity in the spin-glass regime}

A single magnetic transition into a short range antiferromagnetically correlated spin-glass like state is observed for $\mathrm{p}>0.02$ in $\mathrm{La}_{2-x} \mathrm{Sr}_{x} \mathrm{CuO}_{4}$ and $\mathrm{p}>0.035$ in $\mathrm{Y}_{1-x} \mathrm{Ca}_{x} \mathrm{Ba}_{2} \mathrm{Cu}_{3} \mathrm{O}_{6}$. With decreasing temperature, we observe a slowing down of the AF spin fluctuations towards the glass transition temperature, which is defined by the maximum in $1 / \mathrm{T}_{1}$ (corresponding to a correlation time of the spin fluctuations of about $10^{-7} \mathrm{~s}$ ). The spin-glass character of this magnetic state has been demonstrated recently for $\mathrm{La}_{1.96} \mathrm{Sr}_{0.04} \mathrm{CuO}_{4}$ where the susceptibility exhibits irreversible and remnant behavior and obeys scaling laws [16]. $\mathrm{T}_{g}$ is significantly higher presumably due to bilayer interactions in $\mathrm{Y}_{1-x} \mathrm{Ca}_{x} \mathrm{Ba}_{2} \mathrm{Cu}_{3} \mathrm{O}_{6}$ than in $\mathrm{La}_{2-x} \mathrm{Sr}_{x} \mathrm{CuO}_{4}$.

It is remarkable that the average internal field at the muon site is only modestly reduced while the transition temperature is lowered by about one order of magnitude. This is illustrated in Fig. 5b, where we display the zero temperature limit of the internal field at the muon site normalized to its value at zero doping. The width of the field distribution, $\Delta \mathrm{B}_{\mu}$, which is a measure of the degree of disorder of the magnetic state, increases linearly with hole doping in this regime as can be seen in Fig. 5c. The modest reduction of the average internal field and 

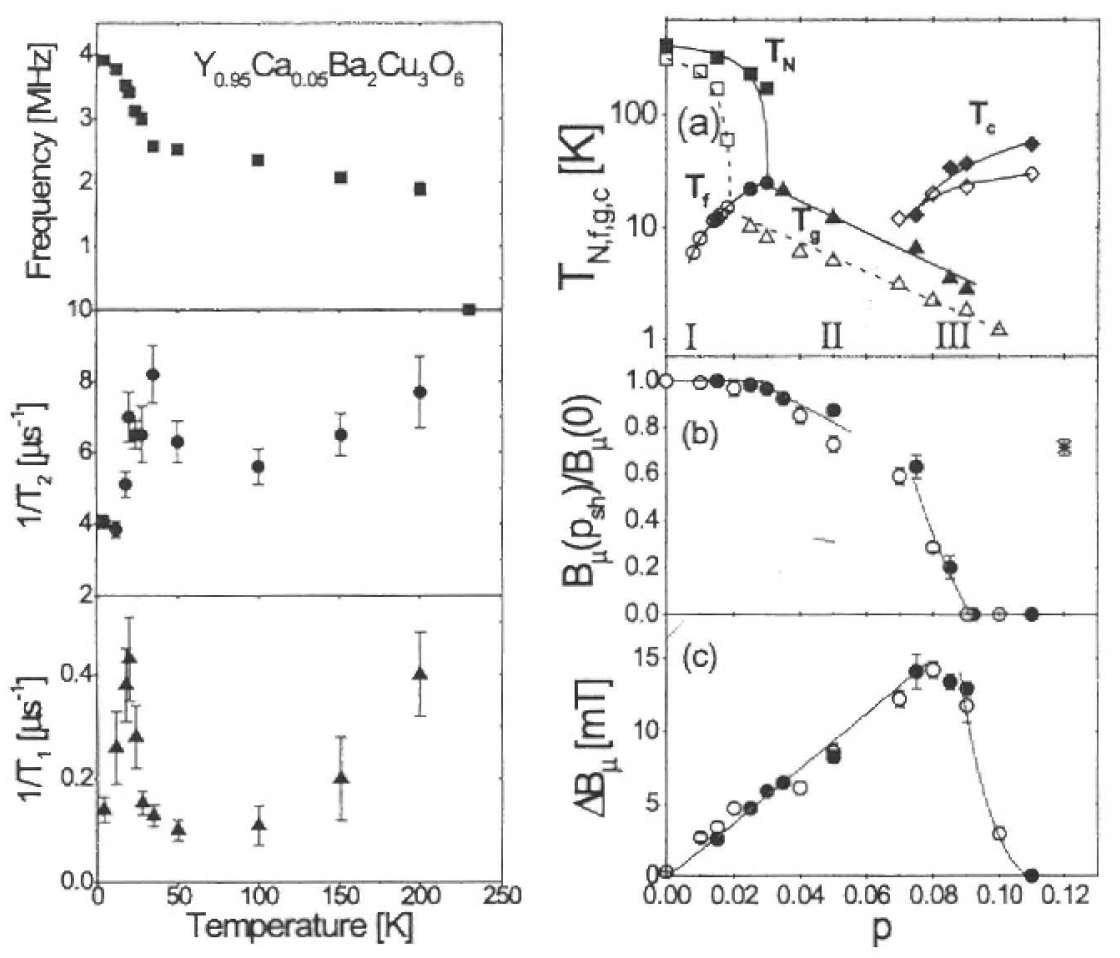

Figure $4 \mathrm{ZF}-\mu \mathrm{SR}$ results on $\mathrm{Y}_{0.95} \mathrm{Ca}_{0.05} \mathrm{Ba}_{2} \mathrm{Cu}_{3} \mathrm{O}_{6}$ plotted as a function of temperature. From top to bottom: Muon spin precession frequency, transverse relaxation rate $1 / T_{2}$ and longitudinal relaxation rate $1 / T_{1}$.

Figure 5 Magnetic phase diagram as a function of the hole concentration per $\mathrm{CuO}_{2}$ sheet for $\mathrm{La}_{2-x} \mathrm{Sr}_{x} \mathrm{CuO}_{4}$ (open symbols) and $\mathrm{Y}_{1-x} \mathrm{Ca}_{x} \mathrm{Ba}_{2} \mathrm{Cu}_{3} \mathrm{O}_{6}$ (full symbols). a) In regime I two transitions are observed. A Néel transition at $T_{N}$ (squares) and a freezing of the spins of the doped holes at $\mathrm{T}_{f}=815 \mathrm{~K} \cdot \mathrm{p}$ (circles, including data from Borsa et al. [13]). $\mathrm{T}_{g}$ indicates a transition into a spin-glass like state (up triangles, regime II) with strong magnetic correlations which coexist with superconductivity in regime III. Diamonds represent the superconducting transition temperatures. b) Doping dependence of the normalized average internal magnetic field at the muon site. The star at $p=0.12$ represents the data for $\mathrm{La}_{1.58} \mathrm{Nd}_{0.3} \mathrm{Sr}_{0.12} \mathrm{CuO}_{4}$. c) rms deviation $\Delta \mathrm{B}_{\mu}$. Data in b) and c) are for $\mathrm{T}<1 \mathrm{~K}$.

the strong increase of $\Delta \mathrm{B}_{\mu}$ with hole doping can be understood in terms of a phase separated electronic state where the holes segregate into metallic domains leaving mesoscopic hole poor regions with antiferromagnetically correlated $\mathrm{Cu}^{2+}$ spins [1].

The spin glass regime extends far into the superconducting state. For strongly underdoped superconducting samples with $0.06<\mathrm{p}<0.10$ we still observe a freezing of the spin degrees of freedom. Except for the somewhat smaller ordering temperature the signature of the transition is the same as for the nonsuperconducting samples. From the amplitude of the rapidly damped muon spin polarization in Fig. 3 we can obtain information about the volume fraction of 
the magnetically correlated regions. We find that all the muons stopped inside the sample experience a non zero local magnetic field, which implies that the magnetic order persists throughout the entire volume of the sample. The magnetic ground state may still be inhomogeneous but the size of the non magnetic hole rich regions must be smaller than the typical length scale (about $2 \mathrm{~nm}$ ) of the $\mu \mathrm{SR}$ experiment. By decoupling experiments in a longitudinal field we have confirmed the static nature of the magnetic ground state.

In contrast to $\mathrm{T}_{g}$ which evolves rather smoothly, the internal magnetic field at the muon site exhibits a strong change for $\mathrm{p} \approx 0.06-0.08$ as one enters the $\mathrm{SC}$ regime. The change in slope is rather significant and indicates a distinct change in the ground state properties of the $\mathrm{CuO}_{2}$ planes. From the $\mu \mathrm{SR}$ experiment alone we can not decide whether it is the competition between the AF and the $\mathrm{SO}$ order parameter or an underlying change of the electronic properties of the $\mathrm{CuO}_{2}$ planes which causes the suppression of the internal field. Further experiments will be required in order to clarify if the SC order parameter is affected by the static AF correlation.

\subsection{Stripe ordering in underdoped cuprates}

Notably, the $A F$ correlation is fully restored at $\mathrm{p} \approx 1 / 8$. A depression of $T_{c}$ at this hole concentration at first appeared to be uniquely present in $\mathrm{La}_{2-x} \mathrm{Ba}_{x} \mathrm{CuO}_{4}$ [17], but recent studies on $\mathrm{La}_{2}{ }_{x} \mathrm{Sr}_{x} \mathrm{CuO}_{4}$ [18] have shown the presence of a shallow cusp at the same doping level and this behavior may also be related to the $60 \mathrm{~K}$ plateau in $\mathrm{YBa}_{2} \mathrm{Cu}_{3} \mathrm{O}_{7-\delta}[19]$. Detailed $\mu \mathrm{SR}$ studies [20] by Luke et al. and Kumagai et al. show that at this doping level static magnetic order is restored at temperatures below $35 \mathrm{~K}$. Tranquada et al. [21] showed that the static order in $\mathrm{La}_{1.6-0.125} \mathrm{Nd}_{0.4} \mathrm{Ba}_{0.125} \mathrm{CuO}_{4}$ comprised a spatial separation of the spin and charge into AF stripes three lattice spacings wide (hole poor) separated by antiphase domain boundaries of one lattice dimension where the doped holes reside on every second site. If we consider a picture in which a stripe phase were to be established through connectivity of the hole doped regions already existing in region II, the averaged internal magnetic field is expected to be $3 / 4$ of the value of the undoped compound. Interestingly, this value is observed for both the $\mathrm{SC}$ compound $\mathrm{La}_{1.93} \mathrm{Sr}_{0.07} \mathrm{CuO}_{4}$ and the non-SC static stripe phase compound $\mathrm{La}_{1}{ }_{58} \mathrm{Nd}_{0.3} \mathrm{Sr}_{0.12} \mathrm{CuO}_{4}$. Moreover, as displayed in Fig. 6, the measured time evolution of the muon spin asymmetry is almost identical for both compounds suggesting the presence of similar local magnetic order.

This is consistent with the suggestion that the incommensurate magnetic peaks seen in $\mathrm{La}_{2-x} \mathrm{Sr}_{x} \mathrm{CuO}_{4}$ over a broad doping range [22] may signal the presence of stripe correlations persisting well away from $1 / 8$ th doping and the possibility that these may be ubiquitous and even fundamental to superconductivity in the cuprates [2]. According to Kivelson and Emery [4] self organization 


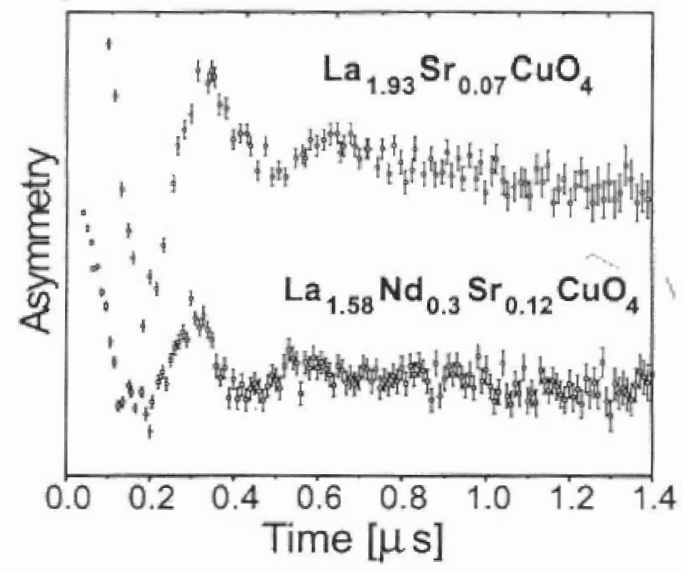

Figure $6 \quad \mathrm{ZF}-\mu \mathrm{SR}$ spectra of $\mathrm{La}_{1.93} \mathrm{Sr}_{0.07} \mathrm{CuO}_{4}$ and $\mathrm{La}_{1.58} \mathrm{Nd}_{0.3} \mathrm{Sr}_{0.12} \mathrm{CuO}_{4}$ for $T=0.1 \mathrm{~K}$ and $\mathrm{T}=10 \mathrm{~K}$, respectively. The latter temperature was chosen to avoid the extra complications associated with the effects of the slowing down of the Nd moments below this temperature. The two spectra are shifted vertically.

into stripes generates a two component electronic system - a localized spin component which lives between the stripes, and a metallic component which flows along the stripes. The spin glass is thus a "cluster spin glass", in which a frozen random array of stripes ("stripe glass") produces the frustration, so that the spin glass consists of patches of locally antiferromagnetically ordered spins with an axis of magnetization that varies from patch to patch.

The antiferromagnetic cluster nature of the spin-glass was very recently microscopically confirmed by other experimentell techniques, ${ }^{63} \mathrm{Cu}$ and ${ }^{139} \mathrm{La} \mathrm{NMR}$ measurements by Julien and coworkers [23] demonstrate the coexistence of spinglass and superconducting phases in $\mathrm{La}_{1.94} \mathrm{Sr}_{0.06} \mathrm{CuO}_{4}$ single crystals. ARPES spectra measured by Ino et al. clearly show the presence of two spectral components coexisting around the superconductor-insulator transition suggesting a microscopic inhomogeneity of the hole density in the sense that the doped holes are segregated in boundaries of antiferromagnetic domains on the scale of several atomic distances [24]. Moreover, Hunt et al. demonstrated that one can measure the charge stripe order by means of ${ }^{63} \mathrm{Cu}$ NQR. Their data reveal the presence of stripe order over the entire underdoped superconducting regime of $\mathrm{La}_{2-x} \mathrm{Sr}_{x} \mathrm{CuO}_{4}[25]$.

The consistency of our results suggests that the coexistence of superconductivity and frozen antiferromagnetic correlations at low temperatures for underdoped samples is an intrinsic property of the $\mathrm{CuO}_{2}$ planes and not an artifact of chemical or structural impurities. Our data show that the strength of the antiferromagnetic correlation is determined solely by the hole content of the $\mathrm{CuO}_{2}$ planes and does not depend on the concentration of dopant atoms, since for a given hole content the number of dopant atoms $\left(\mathrm{Ca}^{2+}\right.$ or $\mathrm{Sr}^{2+}$ ) is twice the number in $\mathrm{Y}_{1-x} \mathrm{Ca}_{x} \mathrm{Ba}_{2} \mathrm{Cu}_{3} \mathrm{O}_{6}$ compared to $\mathrm{La}_{2-x} \mathrm{Sr}_{x} \mathrm{CuO}_{4}$. 
The experiments described herein have been performed at Paul Scherrer Institute, Villigen, Switzerland and at TRIUMF, Vancouver, Canada. We thank these institutions and their support staff for continuing assistance. Funding assistance from the BMBF and the DFG is gratefully acknowledged.

\section{Bibliography}

[1] V.J. Emery and S.A. Kievelson, Physica C 209, 597 (1993).

[2] V.J. Emery, S.A. Kievelson and O. Zachar, Phys. Rev. B 52, 7334 (1995).

[3] S.A. Kievelson, E. Fradkin and V.J. Emery, Nature 393, 550 (1998).

[4] S. A. Kievelson and V. J. Emery, cond-mat/9809082.

[5] For a review of the $\mu \mathrm{SR}$ technique see A. Schenck, Muon Spin Rotation Spectroscopy, (Adam Hilger, Bristol, 1985).

[6] J.L.Tallon, C. Bernhard, Ch. Niedermayer, Supercond. Sci. Technol. 10, A38 (1997).

[7] W.K. Dawson et al., J. Appl. Phys. 64, 5803 (1988); M. Weber at al., Hyp.Int, 63, 207 (1990).

[8] J.I. Budnick et al., Europhys. Lett. 5, 65 (1988); A. Weidinger et al., Phys. Rev. Lett. 62, 102 (1989)

[9] N. Nishida et al., J. Phys. Soc. Jpn 57, 597 (1988); A. Weidinger et al., Hyp. Int. 63,147 (1990).

[10] S.D. Obertelli, J.R. Cooper and J.L. Tallon, Phys. Rev. B 46, 14928 (1992)

[11] J.Tallon, C. Bernhard, and J.D. Jorgensen, to be published.

[12] F.C. Chou et al., Phys. Rev. Lett. 712323 (1993).

[13] F. Borsa et al., Phys. Rev. B 52, 7334 (1995).

[14] R. J. Gooding et al., Phys. Rev. B 49, 6067 (1994).

[15] H. Casalta, H. Alloul and J.-F. Marucco, Physica C 204, 331 (1993).

[16] F. C. Chou et al., Phys. Rev. Lett. 75, 2204 (1995).

[17] A.R. Moodenbaugh et al., Phys. Rev. B 38, 4596 (1988)

[18] P.G. Radaelli et al., Phys. Rev. B 49, 4163 (1994)

[19] J.L. Tallon et. al, Physica C 282-287, 236 (1997)

[20] G.M. Luke et al., Physica C 185-189, 1175 (1991); K. Kumagai et al., Physica C 185-189, 913 (1991).

[21] J.M. Tranquada et al., Nature 375, 561 (1995); J. M. Tranquada et al., Phys. Rev. Let.t. 78, 338(1997).

[22] K. Yamada et al., Phys. Rev. B 57, 6165 (1988).

[23] M.-H. Julien et al., cond-mat/9903005.

[24] A. Ino et al., cond-mat/9902048.

[25] A.W. Hunt et al., cond-mat/9902348. 